\title{
Data modeling for Ambient Home Care Systems
}

\author{
Ana M. Bernardos, M. del Socorro Bernardos, Josué Iglesias, José R. Casar \\ Universidad Politécnica de Madrid, Madrid (Spain) \\ \{ abernardos, josue, jrcasar\}@grpss.ssr.upm.es, sbernardos@fi.upm.es
}

\begin{abstract}
Ambient assisted living (AAL) services are usually designed to work on the assumption that real-time context information about the user and his environment is available. Systems handling acquisition and context inference need to use a versatile data model, expressive and scalable enough to handle complex context and heterogeneous data sources. In this paper, we describe an ontology to be used in a system providing AAL services. The ontology reuses previous ontologies and models the partners in the value chain and their service offering. With our proposal, we aim at having an effective AAL data model, easily adaptable to specific domain needs and services.
\end{abstract}

\section{Introduction}

The population pyramid in many developed countries shows a constrictive profile, due to a lower percentage of young people than some decades ago and an expansive number of elderly and mature citizens, with higher life expectancy but also with more chronic diseases to cope with. This situation is a serious challenge for social (health)care systems, which need to offer high quality services while guaranteeing their economic sustainability. For this reason, technologies to promote independent living are in the research strategic agenda in many countries [1]. In particular, the development of Ambient Care Systems (ACS) are attracting a lot of interest in the research community: enhancing the elder's care network, detecting and handling emergency situations and helping the users to accomplish activities of daily living (eg. fulfilling medication intake schedules) are some of their functional objectives.

Ambient Care Systems are based on an opportunistic and intelligent acquisition of information from heterogeneous sources (mobile phones, personal biometric sensors or infrastructure devices), its subsequent aggregation with static data (such as profile information, personal calendars or electronic health records) and an accurate and real-time inference of information about users' contexts [2]. ACSs are supposed to take decisions on the user's 'context images', triggering events and actions, and offering support to decision processes. At the same time, ACSs need to be configured to serve as channels for third parties to integrate external services, so they need to provide interoperable communications and authentication and 
security features. Then, ACSs are context-aware systems needing to cope with a great diversity of data, which should be sharable among different types of consumer applications with different informational needs. How to represent context parameters in an expressive and extendable way is still an open issue [3], as general data models are not adapted to the ACSs information needs.

A trend in context modeling is to use ontologies to represent context data; due to their versatility in terms of distribution, validation, formalization, ambiguity control and completeness. In this paper we propose an application-oriented ontology (ACS-Ont), specifically designed to cover the representation needs of an ambient care system. ACS-Ont reuses previous ontologies and expands some others, in order to generate a domain data model.

Our proposal differs from previous ones in its holistic approach: ACS-Ont covers the deployment of services both in residential environments and outdoors and considers the integration of different stakeholders in the service provision value chain. ACS-Ont is scalable in terms of sensor and service diversity, and has been built following the reusability paradigm for data modeling, by focusing on the exploitation of existent ontologies.

The paper is structured as follows. Section II reviews the state-of-the-art in ontology-based context modeling, with a special focus on remote healthcare systems. Section III explains the service scenario and its requirements. Section IV covers the ACS-Ont design process and our ontology models reuse strategy. Section V underlines the open issues.

\section{State of the Art}

An ontology is a formal explicit specification of a shared abstract model representing some phenomenon [5]. In practice, an ontology defines a common vocabulary based on the main concepts defining a discourse universe or domain, their properties and the restrictions on the relationships among concepts [6]. Ontologies are powerful tools to specify concepts and relationships, and they simplify interpretation and managing of non-complete data. For these reasons, ontologies have been chosen to model context information (see e.g. [7] [8]). Some examples are: SOUPA [9], CONON [10], the GAIA's context model [12] and CoDAMoS ontology [13]. SOUPA combines a number of useful vocabularies from different ontologies (FOAF, Rei, etc.) to model Person, Policy\&Action, Time or Event. CONON provides an upper ontology to model general concepts and supports extensibility for adding domain ontologies. CONON defines 14 core classes which model Person, Location, Activity and ComputationalEntities. The GAIA architecture uses a context model based on predicates which are afterwards formalized into a DAML+OIL ontology. The ontology facilitates the predicates' consistency checking: e.g. its (XML) description is validated with the ontology when a new entity is included. The particularity of GAIA's proposal is that it aims at modeling 
uncertainty by attaching a confidence value to predicates. CoDAMoS ontology considers the provision of ambient services through a platform, determining four main entities: User, Environment, Platform (hardware and software description of a device) and Service (specific functionalities to the user).

Regarding the needs of ambient care systems, several works have addressed partial aspects of the problem. The K4CARE project [14] proposes a healthcare model for home services which is composed by two ontologies: the Actor Profile Ontology (APO) and the Case Profile Ontology (CPO). This data model neither considers the representation of the infrastructure nor personal sensing devices, nor the service provision. The SOPRANO project [15] is built on an OWL-Lite ontology which only aims at describing the person's state, taking into account past states. Nugent et al. [16] present a XML-based schema representation of information in smart homes. The hierarchical representation describes 1) the home physical structure and the devices in it; 2) the contact details of the institutional caregiver in charge and 3) the inhabitant details (name, ambulatory devices and care plan). In this case, the data model does not consider how to deal with real-time context information. Ko et al. [17] propose the use of a context ontology for UHealthCare. The data model classifies context according to the Person context ontology, Device context ontology and Environment context ontology. In [18], the context model is composed by several OWL ontologies to detect alarms in home environments. The ontology describes the patients, the home domain, the alarm management system and the social care network. Other proposals are focused on the messages exchange system. For example, [19] present a data model for a telemedicine system, which aims at supporting the management of messages exchange between different actors in the telemedicine system. There are also some attempts to model the singularities of special users. It is the case of [20], which proposes a context model to monitor and handle agitation behavior in persons suffering dementia. A great challenge in ambient care systems design is how to make them emotion-aware. With respect to data modeling, ontologies have been also used to model feelings and emotional states (e.g. [17]).

The design of the ACS-Ont has a special focus on reusing existing ontologies while considering the particularities of the ACS domain and its value chain. To the best of our knowledge, none of the previous initiatives completely cover the ACS provision problem.

\section{Model Requirements}

Ambient Care Systems aim at providing different type of assistive services in different scenarios, always taking into account the user's real-time needs. In this Section, our approach to ACS service offering is addressed. From it, we derive the requirements of the data model which will be afterwards implemented in ACS-Ont ontology. Fig. 1 represents our understanding of the service paradigm for ACS. 
The ACS will be working on:

1. A Personal Network (PN), which will include all the devices that a person must wear or use. For instance, localization may be supported by a mobile device with GPS capabilities (for outdoor positioning) or with communication capabilities such as WiFi, Bluetooth and/or ZigBee (for outdoor positioning), or by other means (e.g. RFID-based user-object interactions). Additionally, depending on the user profile and the services to be configured, the PN may include continuous health monitoring sensors (ECG, oxymeters, etc.).

2. A Home Network, which will include home infrastructure sensors, actuators and appliances capable of notifying their status. The Home Network Unit will be able to communicate with the Personal Network by using ad hoc networking capabilities. It will include local intelligent features to dispatch events and orders depending on the situation. These processing capabilities will be part of a home gateway which will connect the home environment with the Core Care Network.

3. A Core Care Network, serving as a bridge of communication between the home infrastructure and third parties and service or context providers. Services may be enabled through the Core Care Network; it will authorize the connection of external service providers (External Care Network) and External Context Data Providers, centralize system monitoring and guarantee the security of personal data.

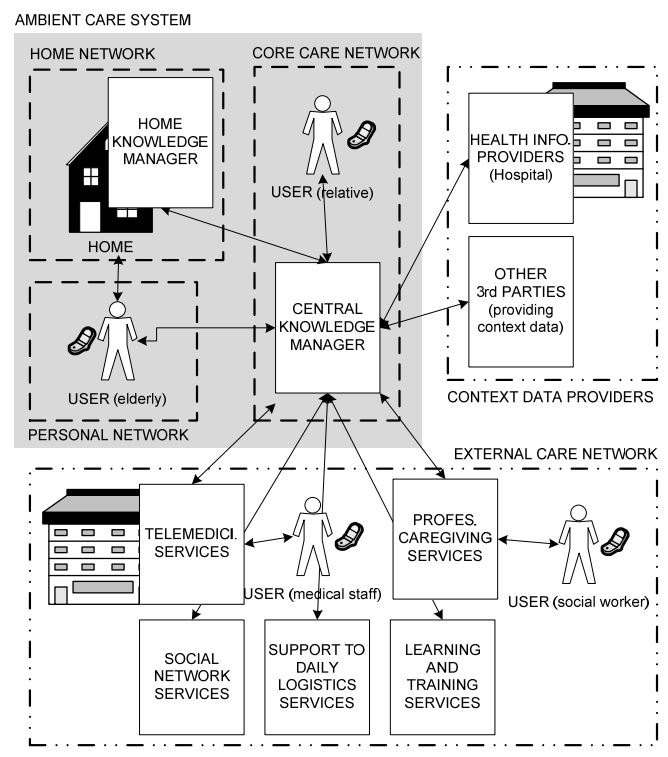

Fig. 1. The service concept behind the Ambient Care System

On top of this structure, the ACS will build its own basic services, such as: a) context-aware notifications/reminders dispatching, b) emergency detection or c) 
activity logging. The first set of services is provided to the elder or to his caregiver, and can be configured to dispatch reminders of medication intake, appointment schedule or general context-aware notifications. The second set of services relies on the processing of biomedical data and environmental information checking to detect risky states. Emergencies are treated both as dangerous situations in the present and long-term deviations from typical behavior that can trigger a complicated situation in the future. Connecting with this case, last group of services stores all the information about activity, allowing an off-line pattern analysis which may feedback the system intelligence.

The system using the ontology will need to:

- Dynamically check consistency when including new concepts.

- Deal with information retrieval and data fusion at different levels of abstraction.

- Support probabilistic inference mechanisms, by handling uncertainty and quality of context.

- Offer a coarse-grained design for general service support, in order to facilitate service scalability.

- Provide data structures to support security: authentication, traceability and data logging.

This operative scenario and the requirements it imposes to a context management system have been taken as the starting point to design ACS-Ont. Next step in the development of the ontology has been the specification of both class representing concepts and relations among them, as explained in the following section.

\section{ACS-Ont structure: methodology, main concepts and sub- ontologies}

We started the development of the ontology by defining its domain and scope. Then we conducted a knowledge acquisition task based on the analysis of different documentation related to the field and on the background of some members of the team. As a result of this process, we could establish the relevant terms of the domain and started to define and (hierarchically) organize the classes of the ontology. We selected the terms that describe objects having independent existence rather than terms that describe these objects.

Due to the large scale of the proposed ACS domain (ranging from specific device and sensor characteristics to concepts related to the users' health status), the domain of interest has been divided into smaller ones: a general ontology regarding the most abstract concepts and relations (those from Fig. 1) has been created and these concepts and relations have been extended generating other ontologies ('sub-ontologies'). The process followed for developing the taxonomies of sub- 
ontologies has been a combination of top-down and bottom-up approaches: first the more salient classes have been defined and then they have been generalized and specialized appropriately.

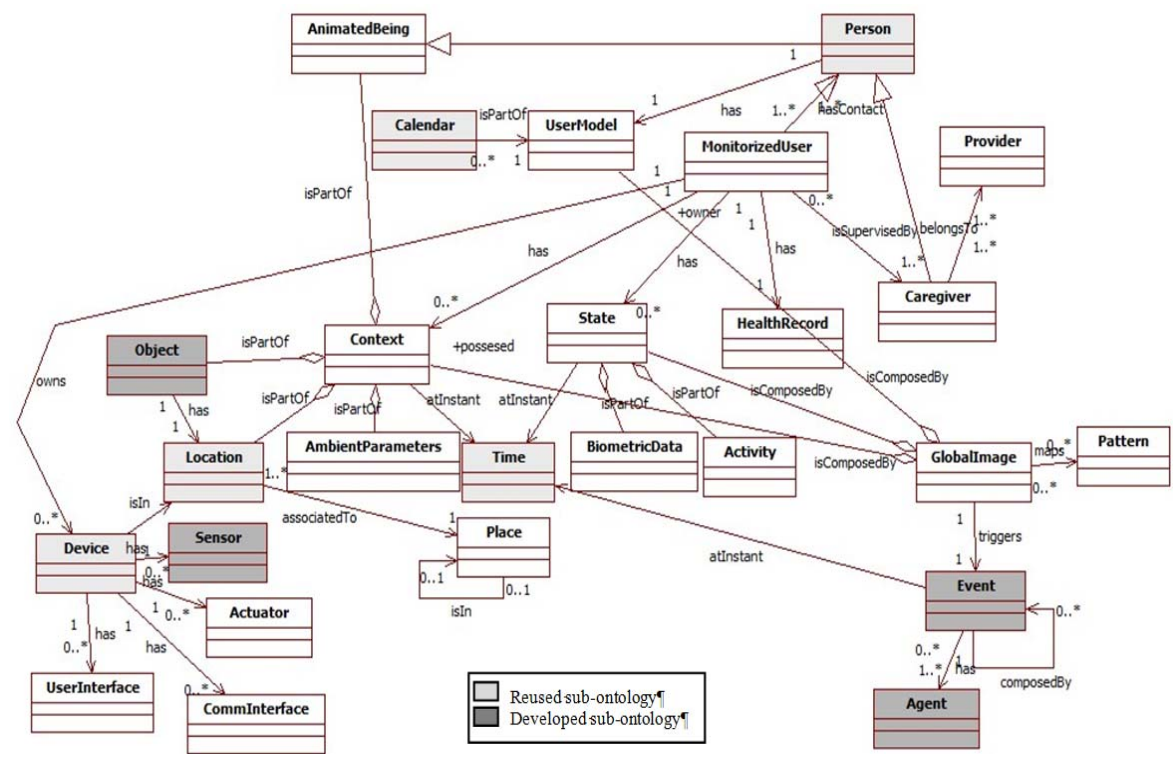

Fig. 2. ACS-Ont structure.

ACS-Ont has been built taking into account that an advantage of ontology models is their capability to be reused. Thus, groups of experts in specific domains might put all their effort into developing solid ontological models of a specific domain that other developers may integrate (as a whole or, more often, just in part) in their own works. This 'share-ability' capability is especially important for highly heterogeneous domains as the present one (with a high diversity of sensor and devices, but also of services and actors). ACS-Ont incorporates different ontologies to describe some common aspects of Ambient Intelligent systems (See Table 1). These models have been directly integrated (either completely or partially) -specially when they are available in the language used for ACS-Ont: OWLor taken just as reference models -in other cases. Color-coded schema of Fig. 2 shows the relations among concepts of general ontology and sub-ontologies, also highlighting reused models.

Some other well-known ontologies have been indentified in order to be reused in future extensions of ACS-Ont (mainly in the development of sub-ontologies). Regarding healthcare operability, an OpenEHR [21] ontology may extend the concepts below HealthRecord class, where SNOMED-CT model [22] can be also integrated (when modeling, for instance, medicines or electronic prescriptions); nevertheless, OWL-based ontologies associated to these models are still an open 
issue. Finally, from a more general view, the ACS security support may be modeled by means of REI Policy Ontology [23] that can be used to formally describe permissions and obligations.

Table 1. Overview of reused ontologies ${ }^{*}$

\begin{tabular}{lll}
\hline Entity/Concept & From ontology & Ontology URI \\
\hline Time & OWL-Time & http://www.isi.edu/ pan/damltime/time-entry.owl \\
Location & Geo-OWL & http://www.opengis.net/gml/ \\
Person & FOAF & http://xmlns.com/foaf/0.1/ \\
Calendar & RDF Calendar & http://www.w3.org/2002/12/cal/icaltzd \\
Device & Delivery Context Ont. & http://www.w3.org/2007/uwa/context/ \\
*General structure of SOUPA, CONON, CODAMOS and X73 models has also been considered.
\end{tabular}

ACS-Ont has been implemented in OWL, fulfilling the OWL-DL expressivity and therefore allowing using any Description Logics reasoner to exploit it.

\section{Conclusions/Discussion and Further Work}

Although this ontology is in a stage of development (the appropriate subontologies have been identified but some of them have not been completely developed yet), it constitutes a headway towards an optimal structuring of the AAL domain. If properly completed, it could be considered as a generic semantic frame for use within this field, since it contributes to the shaping of a common, shared knowledge based in reusability and that can be reused.

A future phase of this work will deal with: a) the enrichment of the ontology by adding possible rules, axioms and constants; b) extending the model to cover specific service needs (e.g. modeling activities of daily living to support behavioral analysis) and c) offering uncertainty control and Quality of Context.

In addition, the use of the application will lead to the introduction of instances that will populate the ontology and allow the application to make inferences or other types of reasoning.

Acknowledgments This work has been supported by the Government of Madrid under grant S2009/TIC-1485 and by the Spanish Ministry of Science and Innovation under grant TIN200806742-C02-01. The authors also acknowledge related discussions with partners within the AmIVital Cenit project, financed by the Centre for the Development of Industrial Technology (CDTI). 


\section{References}

[1] The Ambient Assisted Living (AAL) Joint Programme, http://www.aal-europe.eu/.

[2] Dey A.K, Abowd G. (1999) Towards a Better Understanding of Context and ContextAwareness. Proc. of the 1st international symposium on Handheld and Ubiquitous Computing, Vol. 1707, pp. 304-307.

[3] Bolchini C., et al. (2007) A Data-oriented Survey of Context Models. SIGMOD Rec., Vol. 36, No. 4, pp. 19-26.

[4] Boury-Brisset A.-C., (2003) Ontology-based Approach for Information Fusion, Proc. of the 6th Int. Conference on Information Fusion.

[5] Gruber T. (1995) -Toward Principles for the Design of Ontologies Used for Knowledge Sharing. Int. Journal Human-Computer Studies, Vol. 43, Issues 5-6, November, p.907-928.

[6] Noy N.F., McGuinness D.L. (2005) Desarrollo de Ontologías. http://protege.stanford. edu/publications/ ontology_development/ontology101-es.pdf. Accessed June 2008.

[7] Baumgartner N., Retschitzegger W. (2006) A Survey of Upper Ontologies for Situation Awareness. Proc. of the Int. Conf. on Knowledge Sharing and Collaborative Engineering.

[8] Ye J. et al. (2007) Ontology-based models in pervasive computing systems. The Knowledge Engineering Review, Vol. 22:4, 315-347.

[9] Chen H. et al. (2004) SOUPA: Standard Ontology for Ubiquitous and Pervasive Applications. Proc. First Annual International Conference on Mobile and Ubiquitous Systems (MobiQuitous'04), pp. 258-267.

[10] Wang X. H., Zhang D. Q., Gu T., Pung H. K. (2004) Ontology Based Context Modeling and Reasoning using OWL. Proc. of the 2nd IEEE Annual Conf. on Pervasive Computing and Communications Workshops.

[11] Khedr M., Karmouch A. (2004) Negotiating Context Information in Context-Aware Systems. IEEE Computer, Vol. 19, No. 6, pp. 21-29.

[12] Ranganathan A., et al. (2003) Ontologies in a Pervasive Computing Environment, Proc. of the Workshop on Ontologies and Distributed Systems.

[13] Preuveneers D., et al. (2004) Towards and Extensible Context Ontology for Ambient Intelligence, LCNS 3295, pp. 148-159.

[14] K4CARE: Knowledge-based HomeCare eServices for an Ageing Europe. "Deliverable 01: The K4Care Model” (2006).

[15] Klein M. et al. (2007) Ontology-Centred Design of an Ambient Middleware for Assisted Living: The Case of SOPRANO, 30th Annual German Conf. on Artificial Intelligence.

[16] Nugent C.D. et al. (2007) homeML-An Open Standard for the Exchange of Data Within Smart Environments. LNCS 4551, pp. 121-129.

[17] Ko E. J. et al. (2007) Ontology-Based Context Modeling and Reasoning for U-HealthCare, IEICE Trans. Inf.\&Syst.,Vol. E90-D, No.8.

[18] Paganelli F., Giuli D. (2007) An ontology-based context model for home health monitoring and alerting in chronic patient care networks. Proc. of the 21st Conf. on Advanced Information Networking and Applications Workshops.

[19] Nageba E. et al. (2009) A model driven ontology-based architecture for supporting the quality of services in pervasive telemedice applications, Proc. of Pervasive Health.

[20] Fook V.F.S., Tay S.C., Jayachandran M., Biswas J., Zhang D. (2006) An ontology-based context model in monitoring and handling agitation behaviour for persons with dementia. Proc. IEEE Int. Conf. on Pervasive Computing and Communications Workshops.

[21] openEHR Foundation, http://www.openehr.org/

[22] College of American Pathologists. Snomed Clinical Terms ${ }^{\circledR}$ Technical Reference Guide, July 2003 release.

[23] Lalana Kagal et al. A Policy Based Approach to Security for the Semantic Web. In 2nd International Semantic Web Conference (ISWC2003), September 2003. 